\title{
Monotone Iterative Technique for Partial Dynamic Equations of First Order on Time Scales
}

\author{
Peiguang Wang ${ }^{1}$ and Ping $\mathrm{Li}^{2}$ \\ ${ }^{1}$ College of Electronic and Information Engineering, Hebei University, Baoding 071002, China \\ ${ }^{2}$ Department of Mathematics and Information Technology, Bohai Petroleum Vocational University, \\ Renqiu 062552, China
}

Correspondence should be addressed to Peiguang Wang,pgwang@mail.hbu.edu.cn

Received 24 August 2007; Accepted 31 October 2007

Recommended by Leonid Berezansky

This work is concerned with the monotone iterative technique for partial dynamic equations of first order on time scales and for this purpose, the existence, uniqueness, and comparison results are also established.

Copyright (C) 2008 P. Wang and P. Li. This is an open access article distributed under the Creative Commons Attribution License, which permits unrestricted use, distribution, and reproduction in any medium, provided the original work is properly cited.

\section{Introduction}

The study of dynamic equations on time scales is an area of research that has received a lot of attention. Many authors have been devoted to the qualitative research of ordinary differential equations on time scales. We can refer to monographs by Bohner and Peterson [1], Lakshmikantham et al. [2], and the references cited therein. With the development of technology, in recent years, some authors have explored the partial differential equations on time scales. Ahlbrandt and Morian [3] have introduced definitions of time scale derivatives and integrals for functions of two variables and obtained the results that include an Euler-Lagrange equation for double integral variational problems on time scales and a Picone identity which implies a Sturm-Picone comparison theorem for second-order elliptic partial differential equations on time scales. Jackson [4] has extended the existing ideas of the univariate case of the time scales calculus to the multivariate case.

Lakshmikantham et al. [5] investigated the monotone iterative technique for partial differential equations of first order in 1984. Nevertheless, up till now, we have not yet seen any research about monotone iterative technique for partial dynamic equations on time scales. In this paper, we will investigate the monotone iterative technique for partial dynamic equations of 
first order on time scales. Firstly, in Sections 2 and 3, we will introduce the univariable calculus and multivariable calculus on time scales that will be the preliminary work in order to define the partial dynamic equations on time scales. Secondly, in Sections 4 and 5, we will present existence, uniqueness, and comparison results and provide the monotone iterative technique for such partial dynamic equations.

\section{Univariable calculus on time scales}

For the detail of basic notions concerned with time scales, we refer to $[1,2]$. To meet the requirements in the next sections, we introduce some notions and lemma here.

A time scale is a nonempty closed subset of the real number $\mathbb{R}$, and we denote it by the symbol $\mathbb{T}$. We define the forward and backward jump operators $\sigma, \rho: \mathbb{T} \rightarrow \mathbb{T}$ by

$$
\sigma(t)=\inf \{s \in \mathbb{T} \mid s>t\}, \quad \rho(t)=\sup \{s \in \mathbb{T} \mid s<t\}
$$

(supplemented by $\inf \varnothing=\sup \mathbb{T}$ ). A point $t \in \mathbb{T}$ is called right-scattered, right-dense, leftscattered, left-dense if $\sigma(t)>t, \sigma(t)=t, \rho(t)<t, \rho(t)=t$ hold, respectively. The set $\mathbb{T}^{k}$ is defined to be $\mathbb{T}$ if $\mathbb{T}$ does not have a left-scattered maximum; otherwise it is $\mathbb{T}$ without this left-scattered maximum. The graininess $\mu: \mathbb{T} \rightarrow[0, \infty)$ is defined by $\mu(t)=\sigma(t)-t$.

If $f: \mathbb{T} \rightarrow \mathbb{R}$ is a function and $t \in \mathbb{T}^{k}$, then the "delta-derivative" of $f$ at the point $t$ is defined to be the number $f^{\Delta}(t)$ (provided it exists) with the property that for each $\epsilon>0$ there is a neighborhood $U \subset \mathbb{T}$ of $t$ such that

$$
\left|f(\sigma(t))-f(s)-f^{\Delta}(t)[\sigma(t)-s]\right| \leq \epsilon|\sigma(t)-t| \quad \forall s \in U .
$$

Lemma 2.1 (see [1]). Let $f: \mathbb{R} \rightarrow \mathbb{R}$ be continuously differentiable and suppose that $g: \mathbb{T} \rightarrow \mathbb{R}$ is delta differentiable. Then $f \circ g: \mathbb{T} \rightarrow \mathbb{R}$ is delta differentiable and the formula

$$
(f \circ g)^{\Delta}(t)=\left\{\int_{0}^{1} f^{\prime}\left(g(t)+h \mu(t) g^{\Delta}(t)\right) d h\right\} g^{\Delta}(t)
$$

holds.

\section{Multivariable calculus on time scales}

In this section, we generalize existing ideas of the time scales calculus to the multivariate case. Firstly, consider the product $\mathbb{T}^{n}=\mathbb{T}_{1} \times \mathbb{T}_{2} \times \cdots \times \mathbb{T}_{n}$, where $\mathbb{T}_{i}$ is a time scale for all $1 \leq i \leq n$, so $\mathbb{T}^{n}$ is a time scale too. Then for any $x \in \mathbb{T}^{n}$ with $x=\left(x_{1}, x_{2}, \ldots, x_{n}\right)$, in which $x_{i} \in \mathbb{T}_{i}, 1 \leq i \leq n$, define the following:

(i) the forward jump operator $\sigma: \mathbb{T}^{n} \rightarrow \mathbb{T}^{n}$ by $\sigma(x)=\left(\sigma\left(x_{1}\right), \sigma\left(x_{2}\right), \ldots, \sigma\left(x_{n}\right)\right)$, where $\sigma\left(x_{i}\right)$ represents the forward jump operator of $x_{i} \in \mathbb{T}_{i}$ for all $1 \leq i \leq n$. Hereafter, the forward jump operator of $x_{i} \in \mathbb{T}_{i}$ will be denoted by $\sigma\left(x_{i}\right)=\sigma_{i}(x)$;

(ii) the back jump operator $\rho: \mathbb{T}^{n} \rightarrow \mathbb{T}^{n}$ by $\rho(x)=\left(\rho\left(x_{1}\right), \rho\left(x_{2}\right), \ldots, \rho\left(x_{n}\right)\right)$, where $\rho\left(x_{i}\right)$ represents the backward jump operator of $x_{i} \in \mathbb{T}_{i}$ for all $1 \leq i \leq n$. The backward jump operator of $x_{i} \in \mathbb{T}_{i}$ will be denoted by $\rho\left(x_{i}\right)=\rho_{i}(x)$; 
(iii) the graininess function $\mu: \mathbb{T}^{n} \rightarrow \mathbb{R}^{n}$ by $\mu(x)=\left(\mu\left(x_{1}\right), \mu\left(x_{2}\right), \ldots, \mu\left(x_{n}\right)\right)$, where $\mu\left(x_{i}\right)$ represents the graininess function of $x_{i} \in \mathbb{T}_{i}$ on the time scale $\mathbb{T}_{i}$ for all $1 \leq i \leq n$. Again, from this point on the graininess function of the time scale $\mathbb{T}_{i}$ for $x_{i} \in \mathbb{T}_{i}$ will be denoted by $\mu\left(x_{i}\right)=\mu_{i}(x)$;

(iv) $\mathbb{T}^{k n}=\mathbb{T}_{1}^{k} \times \mathbb{T}_{2}^{k} \cdots \times \mathbb{T}_{n}^{k}$.

Secondly, we introduce the definitions of time-scale derivatives for the function $u(t, x)$, where $t \in \mathbb{T}$ and $x \in \mathbb{T}^{n}$. The forward jump operator $\sigma: \mathbb{T} \rightarrow \mathbb{T}$ and $\tau: \mathbb{T}^{n} \rightarrow \mathbb{T}^{n}$ are defined by $\sigma(t)=\inf \left\{s_{1} \in \mathbb{T} \mid s_{1}>t\right\}$ and $\tau(x)=\inf \left\{s_{2} \in \mathbb{T}^{n} \mid s_{2}>x\right\}$. The graininess $h: \mathbb{T} \rightarrow \mathbb{R}$ is defined as $h(t)=\sigma(t)-t$. The graininess $k: \mathbb{T}^{n} \rightarrow \mathbb{T}^{n}$ is defined as $k(x)=\tau(x)-x$. We will use the notation $u^{\sigma}(t, x)=u(\sigma(t), x), u^{\tau}(t, x)=u(t, \tau(x))$ and $u^{\sigma \tau}(t, x)=u(\sigma(t), \tau(x))$.

Because we will need notation for partial derivatives with respect to time-scale variables $t$ and $x$, we employ lexigraphic ordering for consistency. Let $u^{\Gamma}(t, x)$ denote the time-scale partial derivative with respect to $t$, in order to distinguish for the sign of $\Delta$-derivative in Section 2, let $u^{\Delta_{2}}(t, x)$ denote the time-scale partial derivative with respect to $x$.

The following definitions of these partial derivatives are now given.

Definition 3.1. Let $u$ be a real-valued function on $\mathbb{T} \times \mathbb{T}^{n}$. At $(t, x) \in \mathbb{T}^{k} \times \mathbb{T}^{k n}$, we say that $u$ has a "$\Gamma$ partial derivative" $u^{\Gamma}(t, x)$, with respect to $t$, if for each $\varepsilon>0$, there exists a neighborhood $u_{t}$ (open in the relative topology of $\mathbb{T}$ of $t$ ) such that

$$
\left|u(\sigma(t), x)-u\left(s_{1}, x\right)-u^{\Gamma}(t, x)\left[\sigma(t)-s_{1}\right]\right| \leq \varepsilon\left|\sigma(t)-s_{1}\right|
$$

for all $s_{1} \in \mathcal{U}_{t}$. Similarly, we say that $u$ has a " $\Delta$ partial derivative" denoted by $u^{\Delta_{2}}(t, x) \in \mathbb{R}$, with respect to $x$, if for each $\varepsilon>0$ there exists a neighborhood $u_{x}$ of $x$ such that

$$
\left|u(t, \tau(x))-u\left(t, s_{2}\right)-u^{\Delta_{2}}(t, x)\left[\tau(x)-s_{2}\right]\right| \leq \varepsilon\left|\tau(x)-s_{2}\right|
$$

for all $s_{2} \in \mathcal{U}_{x}$.

Finally, we define the partial derivative of $u(t, x)$ at $x$ with respect to the variable $x_{i}$, where $t \in \mathbb{T}$ and $x=\left(x_{1}, x_{2}, \ldots, x_{n}\right) \in \mathbb{T}^{n}$. Having defined the multivariable calculus as earlier, we set

$$
\begin{aligned}
& u^{\sigma_{i}}(t, x)=u\left(t, x_{1}, x_{2}, \ldots, x_{i-1}, \sigma_{i}(x), x_{i+1}, \ldots, x_{n}\right), \\
& u_{i}^{s_{3}}(t, x)=u\left(t, x_{1}, x_{2}, \ldots, x_{i-1}, s_{3}, x_{i+1}, \ldots, x_{n}\right) .
\end{aligned}
$$

Definition 3.2. Let $u: \mathbb{T} \times \mathbb{T}^{n} \rightarrow \mathbb{R}$ be a real-valued function and let $x=\left(x_{1}, x_{2}, \ldots, x_{n}\right) \in \mathbb{T}^{k n}$. Then define $u^{\Delta_{2}^{i}}(t, x)$ to be the number (provided it exists) with the property that given any $\varepsilon>0$, there exists a neighborhood $\mathbb{U}$ of $x_{i}$, with $\mathbb{U}=\left(x_{i}-\delta, x_{i}+\delta\right) \cap \mathbb{T}_{i}$ for $\delta>0$ such that

$$
\left|\left[u^{\sigma_{i}}(t, x)-u_{i}^{s_{3}}(t, x)\right]-u^{\Delta_{2}^{i}}(t, x)\left[\sigma_{i}(x)-s_{3}\right]\right| \leq \varepsilon\left|\sigma_{i}(x)-s_{3}\right|
$$

for all $s_{3} \in \mathbb{U} \cdot u^{\Delta_{2}^{i}}(t, x)$ is called the partial delta derivative of $u(t, x)$ at $x$ with respect to variable $x_{i}$. 


\section{Existence, uniqueness, and comparison result}

In this section, we consider the initial value problem (IVP) for partial dynamic equation of first order on time scales

$$
u^{\Gamma}+f(t, x) u^{\Delta_{2}}=g(t, x, u), \quad u(0, x)=\phi(x)
$$

where $f \in C\left(\Omega, \mathbb{R}^{n}\right), g \in C(\Omega \times \mathbb{R}, \mathbb{R}), \Omega=\left\{(t, x): 0 \leq t \leq T, a \leq x \leq b, t \in \mathbb{T}^{k}, a, b, x \in \mathbb{T}^{k n}\right\}$, $f u^{\Delta_{2}}=\sum_{i=1}^{n} f_{i}(t, x) u^{\Delta_{2}^{i}}$ and $\phi \in C^{1}([a, b], \mathbb{R})$.

We define the set $\mathbb{D}=\left\{u \in C\left[\Omega_{1}, \mathbb{R}\right], u^{\Gamma}, u^{\Delta_{2}}\right.$, and $u^{\Delta_{2}^{i}}$ are continuous on $\left.\mathbb{T}^{k} \times \mathbb{T}^{k n}\right\}, \Omega_{1}=$ $\left\{(t, x), 0 \leq t \leq T, a \leq x \leq \sigma(b), t \in \mathbb{T}^{k}, a, b\right.$, and $\left.x \in \mathbb{T}^{k n}\right\}$.

Now, we begin to prove the following comparison result.

Theorem 4.1. Assume that

$\left(\mathrm{A}_{0}\right) \alpha^{\Gamma}+f(t, x) \alpha^{\Delta_{2}} \leq g(t, x, \alpha), \alpha(0, x) \leq \phi(x)$, and $\beta^{\Gamma}+f(t, x) \beta^{\Delta_{2}} \geq g(t, x, \beta), \beta(0, x) \geq \phi(x)$ for $(t, x) \in \Omega_{1}$, where $\alpha, \beta \in \mathbb{D}$.

$\left(\mathrm{A}_{1}\right) f(t, x)$ is quasimonotone nonincreasing in $x$ for each $i$ and $f(t, a) \leq 0, f(t, b) \geq 0$.

$\left(\mathrm{A}_{2}\right) g\left(t, x, u_{1}\right)-g\left(t, x, u_{2}\right) \leq L\left(u_{1}-u_{2}\right)$ whenever $u_{1} \geq u_{2}$ for some $L \geq 0$.

Then $\alpha(t, x) \leq \beta(t, x)$ on $\Omega_{1}$.

Proof. Let us prove the theorem for strict inequalities firstly. For example, we suppose that $\alpha^{\Gamma}+f(t, x) \alpha^{\Delta_{2}}<g(t, x, \alpha)$ and $\alpha(0, x)<\phi(x)$ on $\Omega_{1}$, and prove that $\alpha(t, x)<\beta(t, x)$ on $\Omega_{1}$. If this conclusion is not true, then consider the set

$$
\mathbb{Z}=\left\{(t, x) \in \Omega_{1} \mid \alpha(t, x) \geq \beta(t, x)\right\}
$$

Let $\mathbb{Z}_{t}$ be the projection of $\mathbb{Z}$ on the $t$-axis and let $t_{0}=\inf \mathbb{Z}_{t}$. Clearly, $t_{0}>0$ and there exists an $x_{0} \in[a, \sigma(b)]$ such that

$$
\begin{aligned}
& \alpha\left(t_{0}, x_{0}\right)=\beta\left(t_{0}, x_{0}\right), \quad \alpha\left(\sigma\left(t_{0}\right), x_{0}\right) \geq \beta\left(\sigma\left(t_{0}\right), x_{0}\right), \\
& \alpha\left(t_{0}, x\right) \leq \beta\left(t_{0}, x\right) \text { for } a \leq x \leq \sigma(b) .
\end{aligned}
$$

It then follows that $\alpha^{\Gamma}\left(t_{0}, x_{0}\right) \geq \beta^{\Gamma}\left(t_{0}, x_{0}\right)$ and if $a \leq x \leq \sigma(b)$, we also have $\alpha^{\Delta_{2}^{i}}\left(t_{0}, x_{0}\right)=$ $\beta^{\Delta_{2}^{i}}\left(t_{0}, x_{0}\right), i=1,2, \ldots, n$. In this case, we get the following contradiction:

$$
g\left(t_{0}, x_{0}, \alpha\left(t_{0}, x_{0}\right)\right)>\alpha^{\Gamma}+f\left(t_{0}, x_{0}\right) \alpha^{\Delta_{2}} \geq \beta^{\Gamma}+f\left(t_{0}, x_{0}\right) \beta^{\Delta_{2}} \geq g\left(t_{0}, x_{0}, \beta\left(t_{0}, x_{0}\right)\right) .
$$

If, on the other hand, for some $j, x_{0, j}=b_{j}$ and $x_{0, i}<b_{i}, i \neq j$, and $a<x_{0}$, then we have $\alpha^{\Delta_{2}^{i}}\left(t_{0}, x_{0}\right)=\beta^{\Delta_{2}^{i}}\left(t_{0}, x_{0}\right), i \neq j$, and $\alpha\left(t_{0}, \sigma\left(x_{0}\right)\right) \geq \beta\left(t_{0}, \sigma\left(x_{0}\right)\right)$, then we have $\alpha^{\Delta_{2}^{j}}\left(t_{0}, x_{0}\right) \geq$ $\beta^{\Delta_{2}^{j}}\left(t_{0}, x_{0}\right)$. Hence using the assumption $\left(\mathrm{A}_{1}\right)$, we obtain $f_{j}\left(t_{0}, x_{0}\right) \geq f_{j}\left(t_{0}, b\right) \geq 0$ and $f_{j}\left(t_{0}, x_{0}\right) \alpha^{\Delta_{2}^{j}}\left(t_{0}, x_{0}\right) \geq f_{j}\left(t_{0}, x_{0}\right) \beta^{\Delta_{2}^{j}}\left(t_{0}, x_{0}\right)$. Consequently, we get the inequality

$$
g\left(t_{0}, x_{0}, \alpha\left(t_{0}, x_{0}\right)\right)>\alpha^{\Gamma}+f\left(t_{0}, x_{0}\right) \alpha^{\Delta_{2}} \geq \beta^{\Gamma}+f\left(t_{0}, x_{0}\right) \beta^{\Delta_{2}} \geq g\left(t_{0}, x_{0}, \beta\left(t_{0}, x_{0}\right)\right),
$$


which is a contradiction as before. This proves that $\alpha(t, x)<\beta(t, x)$ on $\Omega_{1}$. If one of the inequalities in $\left(\mathrm{A}_{0}\right)$ is not strict, and on one hand, if $\mu(t)=0$, we set $\widetilde{\alpha}(t, x)=\alpha(t, x)-\varepsilon e^{2 L t}$ and note that $\tilde{\alpha}<\alpha$. Then using $\left(\mathrm{A}_{0}\right)$ and $\left(\mathrm{A}_{2}\right)$, we have

$$
\begin{aligned}
\tilde{\alpha}^{\Gamma}+f(t, x) \tilde{\alpha}^{\Delta_{2}} & =\alpha^{\Gamma}+f(t, x) \alpha^{\Delta_{2}}-2 L \varepsilon e^{2 L t} \\
& \leq g(t, x, \alpha)-2 L \varepsilon e^{2 L t} \leq g(t, x, \tilde{\alpha})+L \varepsilon e^{2 L t}-2 L \varepsilon e^{2 L t}<g(t, x, \tilde{\alpha}),
\end{aligned}
$$

and $\tilde{\alpha}(0, x)<\phi(x)$ on $\Omega_{1}$. On the other hand, if $\mu(t) \neq 0$, we set $\tilde{\alpha}(t, x)=\alpha(t, x)-\varepsilon e^{L t}$ and note that $\tilde{\alpha}<\alpha$. Then using $\left(\mathrm{A}_{0}\right),\left(\mathrm{A}_{2}\right)$, and Lemma 2.1, we have

$$
\begin{aligned}
\tilde{\alpha}^{\Gamma}+f(t, x) \tilde{\alpha}^{\Delta_{2}} & =\alpha^{\Gamma}+f(t, x) \alpha^{\Delta_{2}}-\varepsilon\left(\frac{e^{L t}}{\mu}\left(e^{L \mu}-1\right)\right) \\
& \leq g(t, x, \alpha)-\frac{\varepsilon e^{L t}}{\mu}\left(e^{L \mu}-1\right) \leq g(t, x, \tilde{\alpha})+L \varepsilon e^{L t}-\frac{\varepsilon e^{L t}}{\mu}\left(e^{L \mu}-1\right) \\
& =g(t, x, \tilde{\alpha})+\frac{L \mu+1-e^{L \mu}}{\mu} \varepsilon e^{L t}<g(t, x, \tilde{\alpha}),
\end{aligned}
$$

and $\tilde{\alpha}(0, x)<\phi(x)$ on $\Omega_{1}$. Thus the foregoing arguments imply that $\alpha(t, x)<\beta(t, x)$ on $\Omega_{1}$. Taking limit as $\varepsilon \rightarrow 0$, we then get $\alpha(t, x) \leq \beta(t, x)$ on $\Omega_{1}$, and the proof is complete.

Theorem 4.2. Assume that $\left(A_{1}\right)$ and $\left(A_{2}\right)$ hold. Suppose further that $\left(A_{3}\right)$ for each $\left(t_{0}, x_{0}\right) \in \Omega$, there exists a unique solution $x\left(t_{0}, x_{0}\right)$ of

$$
x^{\Delta}=f(t, x), \quad x\left(t_{0}\right)=x_{0},
$$

on $0 \leq t \leq T, x\left(t, t_{0}, x_{0}\right)$ is continuously differentiable with respect to $\left(t_{0}, x_{0}\right)$. Assume $\left(A_{4}\right)$ for each $x_{0} \in[a, b]$ and $y_{0} \in \mathbb{R}$, there exists a unique solution $y\left(t, 0, y_{0} ; x_{0}\right)$ of

$$
y^{\Delta}=g\left(t, x\left(t, 0, x_{0}\right), y\right), \quad y(0)=y_{0},
$$

on $0 \leq t \leq T$, where $x\left(t, 0, x_{0}\right)$ is the unique solution of $(4.8)$ and $y\left(t, 0, y_{0} ; x_{0}\right)$ is continuously differentiable with respect to $\left(y_{0}, x_{0}\right)$. on $\Omega_{1}$.

Then there exists a unique solution $u(t, x)=y(t, 0, \phi(x(0, t, x)) ; x(0, t, x))$ for the problem (4.1)

Proof. By $\left(\mathrm{A}_{3}\right)$ and $\left(\mathrm{A}_{4}\right), x\left(t, t_{0}, x_{0}\right), y\left(t, 0, y_{0} ; x_{0}\right)$ are unique solutions of (4.8) and (4.9), respectively, on $0 \leq t \leq T$. Choose $y_{0}=\phi\left(x_{0}\right)$ and note that if $x=x\left(t, 0, x_{0}\right)$, then because of uniqueness, $x_{0}=x(0, t, x)$. Hence, $y(t, 0, \phi(x(0, t, x)) ; x(0, t, x))$ is a unique solution of (4.9). Then we have

$$
y^{\Delta}=y^{\Gamma}+y^{\Delta_{2}} x^{\Delta}=y^{\Gamma}+f(t, x) y^{\Delta_{2}}=g(t, x, y), \quad y(0, x)=\phi(x),
$$

that is, $u(t, x)=y(t, 0, \phi(x(0, t, x)) ; x(0, t, x))$ satisfies (4.1) and consequently, $u(t, x)$ is a solution of the problem (4.1).

To show uniqueness of solution of (4.1), suppose that $u_{1}(t, x), u_{2}(t, x)$ are two solutions of (4.1) on $\Omega_{1}$. Then setting $\alpha=u_{1}, \beta=u_{2}$ and applying Theorem 4.1 , we get $u_{1}(t, x) \leq u_{2}(t, x)$ on $\Omega_{1}$. Similarly we can prove that $u_{1}(t, x) \geq u_{2}(t, x)$ on $\Omega_{1}$. Hence the proof is complete. 


\section{Monotone iterative technique}

we are now in a position to describe the monotone iterative technique which yields monotone sequences on time scales. We prove the following result specifically.

Theorem 5.1. Assume that $\left(A_{0}\right),\left(A_{1}\right)$, and $\left(A_{3}\right)$ hold with $\alpha \leq \beta$ on $\Omega_{1}$. Suppose further $\left(A_{5}\right)$ for some $M>0, g\left(t, x, u_{1}\right)-g\left(t, x, u_{2}\right) \geq-M\left(u_{1}-u_{2}\right)$ whenever $\alpha \leq u_{2} \leq u_{1} \leq \beta$ on $\Omega_{1}$. Assume $\left(A_{6}\right)$ for each $x_{0} \in[a, b]$ and $y_{0} \in \mathbb{R}$, there exists a unique solution $y\left(t, 0, y_{0} ; x_{0}\right)$ of

$$
y^{\Delta}=g\left(t, x\left(t, 0, x_{0}\right), y ; \eta\left(t, x\left(t, 0, x_{0}\right)\right)\right)-M(y-\eta), \quad y(0)=y_{0}
$$

whenever $\alpha \leq \eta \leq \beta$.

Then there exist monotone sequences $\left\{\alpha_{n}(t, x)\right\},\left\{\beta_{n}(t, x)\right\}$, and the functions $\rho(t, x), r(t, x)$ such that if $u$ is any solution of (4.1), then

$$
\alpha \leq \alpha_{1} \leq \cdots \leq \alpha_{n} \leq \rho \leq u \leq r \leq \beta_{n} \leq \cdots \leq \beta_{1} \leq \beta \quad \text { on } \Omega_{1} .
$$

Proof. Consider the linear IVP

$$
u^{\Gamma}+f(t, x) u^{\Delta_{2}}=G(t, x, u ; \eta), \quad u(0, x)=\phi(x),
$$

where $G(t, x, u ; \eta)=g(t, x, \eta)-M(u-\eta)$ and $\eta \in C\left(\Omega_{1}, \mathbb{R}\right)$ is such that $\alpha \leq \eta \leq \beta$ on $\Omega_{1}$.

By $\left(\mathrm{A}_{5}\right)$, we have

$$
g(t, x, \alpha)-G(t, x, \alpha ; \eta)=g(t, x, \alpha)-g(t, x, \eta)+M(u-\eta) \leq M(\eta-\alpha)+M(\alpha-\eta)=0,
$$

so it follows that $\alpha^{\Gamma}+f(t, x) \alpha^{\Delta_{2}} \leq G(t, x, \alpha ; \eta), \alpha(0, x) \leq \phi(x)$ on $\Omega_{1}$. Similarly, we obtain that $\beta^{\Gamma}+f(t, x) \beta^{\Delta_{2}} \leq G(t, x, \beta ; \eta), \beta(0, x) \leq \phi(x)$ on $\Omega_{1}$. Hence $\left(\mathrm{A}_{0}\right)$ holds. Also, if $u_{1} \geq u_{2}$,

$$
G\left(t, x, u_{1} ; \eta\right)-G\left(t, x, u_{2} ; \eta\right)=-M\left(u_{1}-u_{2}\right) \leq L\left(u_{1}-u_{2}\right),
$$

and therefore $\left(A_{2}\right)$ is satisfied for $G$. Furthermore, by $\left(A_{6}\right),\left(A_{4}\right)$ is satisfied relative to

$$
y^{\Delta}=G\left(t, x\left(t, 0, x_{0}\right), y ; \eta\left(t, x\left(t, 0, x_{0}\right)\right)\right), \quad y(0)=y_{0} .
$$

As a result, by Theorem 4.2, there exists a unique solution $u(t, x)$ of (5.3) on $\Omega_{1}$ for every $\eta \in$ $C\left(\Omega_{1}, \mathbb{R}\right)$ such that $\alpha \leq \eta \leq \beta$ on $\Omega_{1}$.

Defining a mapping $A$ by $A \eta=u$, where $u$ is the unique solution of (5.3) corresponding to $\eta$. Concerning this mapping $A$, we will show that (i) $\alpha \leq A \alpha, \beta \geq A \beta$, and (ii) $A$ is monotone on the sector $[\alpha, \beta]$, namely, if $\alpha \leq \eta_{1} \leq \eta_{2} \leq \beta$, then $A \eta_{1} \leq A \eta_{2}$.

Let $\eta=\alpha$ and let $A \alpha=\alpha_{1}$, where $\alpha_{1}$ is the unique solution of (5.3). Then we have $\alpha^{\Gamma}+f(t, x) \alpha^{\Delta_{2}} \leq G(t, x, \alpha ; \alpha), \alpha(0, x) \leq \phi(x)$, and $\alpha_{1}^{\Gamma}+f(t, x) \alpha_{1}^{\Delta_{2}}=G(t, x, \alpha ; \alpha), \alpha_{1}(0, x)=\phi(x)$ on $\Omega_{1}$. By Theorem 4.1, we see that $\alpha \leq \alpha_{1}=A \alpha$. Similarly, we can show that $\beta \geq A \beta$.

To prove (ii), let $\eta_{1}, \eta_{2} \in C\left[\Omega_{1}, \mathbb{R}\right]$ be such that $\alpha \leq \eta_{1} \leq \eta_{2} \leq \beta$ and let $A \eta_{1}=u_{1}, A \eta_{2}=u_{2}$, where $u_{1}, u_{2}$ are the unique solutions of (5.3) corresponding to $\eta=\eta_{1}, \eta=\eta_{2}$, respectively. Then, $u_{1}^{\Gamma}+f(t, x) u_{1}^{\Delta_{2}}=G\left(t, x, u_{1} ; \eta_{1}\right) \leq G\left(t, x, u_{1} ; \eta_{2}\right)$ and $u_{2}^{\Gamma}+f(t, x) u_{2}^{\Delta_{2}}=G\left(t, x, u_{2} ; \eta_{2}\right)$. Also, $u_{1}(0, x)=u_{2}(0, x)$. Hence by Theorem 4.1 , we have $u_{1} \leq u_{2}$ on $\Omega_{1}$. This proves $A \eta_{1} \leq A \eta_{2}$. 
Consider the sequence $\left\{\alpha_{n}(t, x)\right\}$ and note that $\alpha_{n}(t, x)=y_{n}[t, 0, \phi(x(0, t, x)) ; x(0, t, x)]$ on $\Omega_{1}$, where $y_{n}=y_{n}\left(t, 0, y_{0} ; x_{0}\right)$ is the unique solution of (5.6) such that $y_{0}=\phi\left(x_{0}\right)$. Thus $\alpha_{n}\left(t, x\left(t, 0, x_{0}\right)\right)=y_{n}\left(t, 0, \phi\left(x_{0}\right) ; x_{0}\right)$ and $\alpha \leq y_{n} \leq \beta$. Since $\left\{y_{n}\right\}$ is monotone sequence, it is easy to conclude that $y_{n}\left(t, 0, \phi\left(x_{0}\right) ; x_{0}\right)$ converges uniformly and monotonically as $n \rightarrow \infty$. Suppose that $\lim _{n \rightarrow \infty} y_{n}\left(t, 0, \phi\left(x_{0}\right) ; x_{0}\right)=y\left(t, 0, \phi\left(x_{0}\right) ; x_{0}\right)$ on $0 \leq t \leq T$. Then it is clear that $y^{\Delta}\left(t, 0, \phi\left(x_{0}\right) ; x_{0}\right)=g\left(t, x\left(t, 0, x_{0}\right), y\left(t, 0, \phi\left(x_{0}\right)\right) ; x_{0}\right), y(0)=\phi\left(x_{0}\right)$. Consequently, we can now define $\rho(t, x)=y(t, 0, \phi(x(0, t, x)) ; x(0, t, x))$ on $\Omega_{1}$. Similar arguments hold relative to the sequence $\left\{\beta_{n}(t, x)\right\}$ and one defines $r(t, x)=\tilde{y}(t, 0, \phi(x(0, t, x)) ; x(0, t, x))$ on $\Omega_{1}$.

Finally, we show that $\alpha \leq \rho \leq u \leq r \leq \beta$ on $\Omega_{1}$, where $u$ is any solution of (4.1) such that $\alpha \leq u \leq \beta$ on $\Omega_{1}$. For this, it is enough to show that $\alpha_{n} \leq u \leq \beta_{n}$ on $\Omega_{1}$ and this we do by induction. Suppose that $\alpha_{k} \leq u \leq \beta_{k}$ for some $k$ on $\Omega_{1}$. Then we have

$$
\begin{gathered}
\alpha_{k+1}^{\Gamma}+f(t, x) \alpha_{k+1}^{\Delta_{2}}=G\left(t, x, \alpha_{k+1} ; \alpha_{k}\right), \quad \alpha_{k+1}(0, x)=\phi(x), \\
u^{\Gamma}+f(t, x) u^{\Delta_{2}}=g(t, x, u) \geq G\left(t, x, u ; \alpha_{k}\right), \quad u(0, x)=\phi(x) .
\end{gathered}
$$

By Theorem 4.1, we have $\alpha_{k+1} \leq u$ on $\Omega_{1}$. We can show similarly that $u \leq \beta_{k+1}$. Hence it follows that $\alpha_{k} \leq u \leq \beta_{k}$ on $\Omega_{1}$ for all $n$, which prove the claim.

Remark 5.2. We note that if $\left(\mathrm{A}_{4}\right)$ holds, then $\rho$ and $r$ are actually solutions of (4.1).

\section{Acknowledgments}

This work is supported by the Key Project of Chinese Ministry of Education (207014) and the Natural Science Foundation of Hebei Province of China (A2006000941).

\section{References}

[1] M. Bohner and A. Peterson, Dynamic Equations on Time Scales: An Introduction with Applications, Birkhäuser, Boston, Mass, USA, 2001.

[2] V. Lakshmikantham, S. Sivasundaram, and B. Kaymakcalan, Dynamic Systems on Measure Chains, vol. 370 of Mathematics and Its Applications, Kluwer Academic, Dordrecht, The Netherlands, 1996.

[3] C. D. Ahlbrandt and C. Morian, "Partial differential equations on time scales," Journal of Computational and Applied Mathematics, vol. 141, no. 1-2, pp. 35-55, 2002.

[4] B. Jackson, "Partial dynamic equations on time scales," Journal of Computational and Applied Mathematics, vol. 186, no. 2, pp. 391-415, 2006.

[5] V. Lakshmikantham, M. N. Oguztöreli, and A. S. Vatsala, "Monotone iterative technique for partial differential equations of first order," Journal of Mathematical Analysis and Applications, vol. 102, no. 2, pp. 393-398, 1984. 


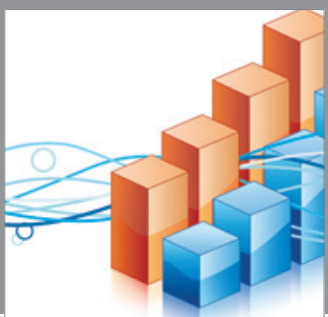

Advances in

Operations Research

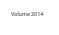

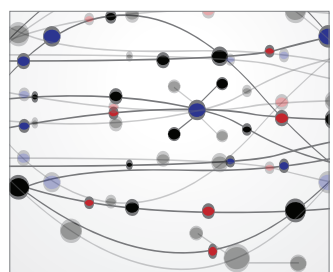

\section{The Scientific} World Journal
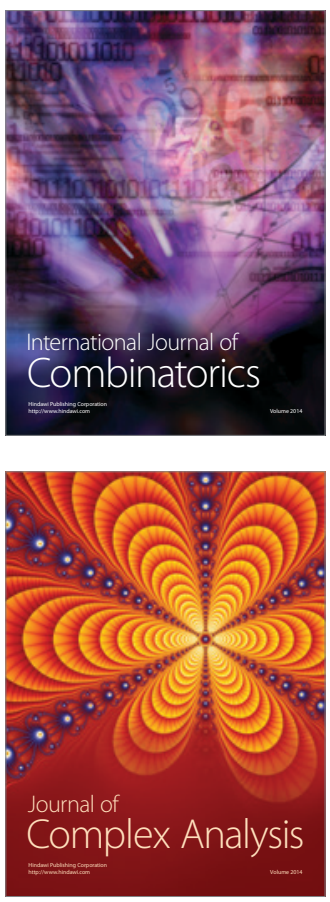

International Journal of

Mathematics and

Mathematical

Sciences
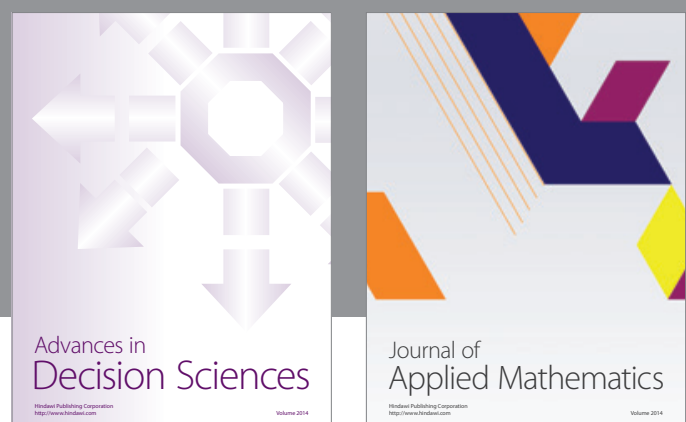

Journal of

Applied Mathematics
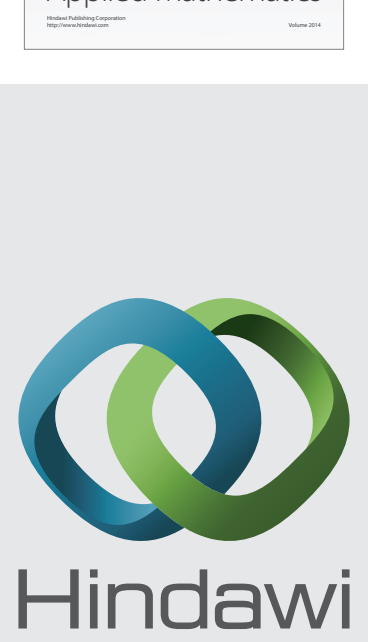

Submit your manuscripts at http://www.hindawi.com
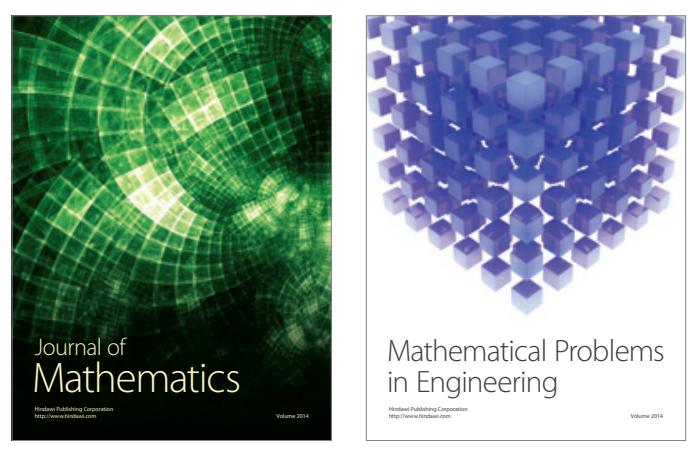

Mathematical Problems in Engineering
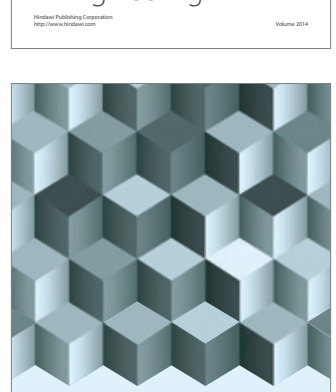

Journal of

Function Spaces
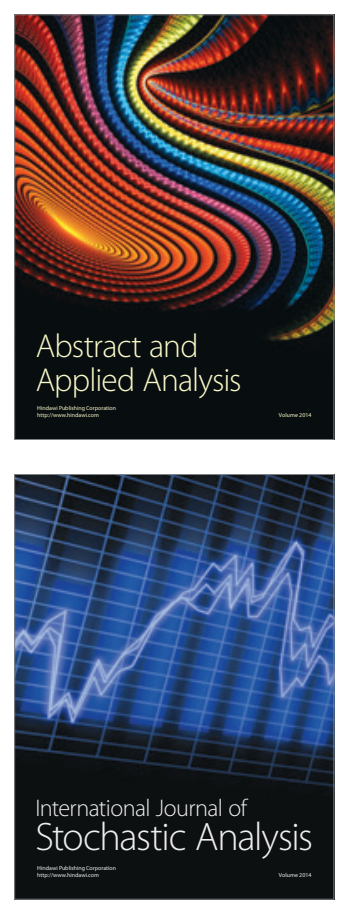

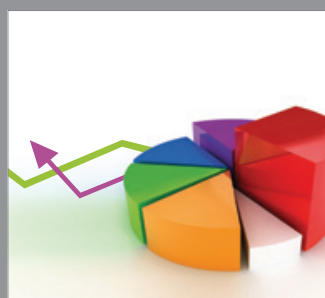

ournal of

Probability and Statistics

Promensencen
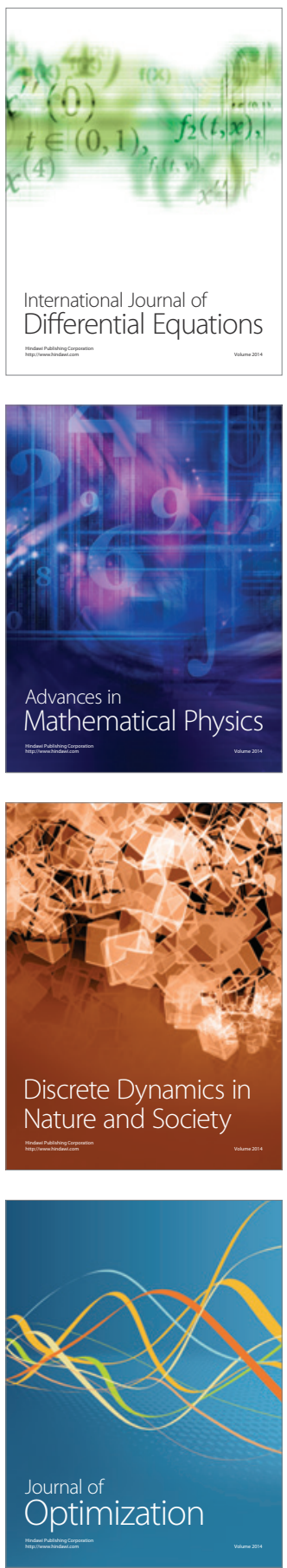